\title{
Informe pericial psicológico en tribunales de familia: análisis de su estructura, metodología y contenido
}

\section{Expert psychological reports in family courts: Evaluating their structure, methodology, and content}

\author{
Carles Rodríguez-Domínguez ${ }^{1}$, Adolfo Jarne Espacia ${ }^{2}$ y Xavier Carbonell ${ }^{1}$ \\ ${ }^{1}$ FPCEE Blanquerna. Universidad Ramón Llull, España. \\ ${ }^{2}$ Universidad de Barcelona, España.
}

Disponible online 30 de abril de 2015

\begin{abstract}
En España, el divorcio contencioso con menores involucrados es frecuente e implica un incremento de demandas de informes psicológicos periciales. El artículo presenta el estudio de la estructura, metodología y contenido en los informes psicológicos aportados a los tribunales de familia, por peritos privados y peritos del equipo psicosocial de los Juzgados de Familia. El objetivo fue analizar su estructura formal, características demográficas y profesionales, metodología, contenidos de la evaluación, garantías y limitación de los resultados, valoración del profesional sobre las afirmaciones del informante en las entrevistas, recomendaciones, y pruebas psicométricas utilizadas así como diferencias y similitudes entre los informes privados y los de los equipos psicosociales. Se administró un plantilla confeccionada por los autores a 111 expedientes contenciosos de juzgados de familia. Los resultados mostraron diferencias significativas en los contenidos de la estructuración, metodología, evaluación y psicometría entre los informes privados y los informes de los equipos psicosociales. Los dictámenes se realizaron mayoritariamente por profesionales de sexo femenino, con buena preparación específica, bien estructurados incluyendo la recomendación del tipo de custodia y régimen de contacto con el progenitor no custodio, la valoración de la competencia parental y la relación de los menores con sus familiares.
\end{abstract}

Palabras Clave: Informe Psicológico; Divorcio Conflictivo; Guarda y Custodia de Menores.

\begin{abstract}
In Spain, contested divorces involving children are frequent and entail an increased demand for expert psychological reports. This article describes the structure, methodology, and content of psychological reports provided to family courts by private expert psychologists and the family court's expert psychosocial team. The aim was to analyse the formal structure of the reports, their demographic and professional characteristics, methodology, assessment content, guarantees and limitations of the results, professional evaluation of the informant's statements during interviews, recommendations, and psychometric tests used. The reports from the private expert psychologists and those from the expert psychosocial teams were compared and contrasted. A template constructed by the authors was used to analyse 111 family court records of contested divorces. The results showed significant differences between the two types of report in their structure, methodology, assessment content, and psychometric tests. Highly qualified women provided the majority of the expert opinions. The reports were well-structured and included recommendations on the type of custody and custody regime for the non-custodial parent, the evaluation of parental competence, and the relationship of the children with their families.
\end{abstract}

Key Words: Psychological Report; Contested Divorce; Guardianship and Custody of Minors.

Correspondencia: Carles Rodríguez Domínguez. FPCEE. c/ Císter 34, Blanquerna. 08022- Barcelona. E-mail: carlesrd@blanquerna.url.edu. E-mails de los co-autores: Adolfo Jarne Espacia: ajarne@ub.edu. Xavier Carbonell: xavics@blanquerna.url.edu

Los autores quieren expresar su agradecimiento al Tribunal Superior de Justicia de Catalunya, y a los Secretarios Judiciales de los Juzgados de Familia, $\mathrm{n}^{\mathrm{o}} 15,16,17,18,19,45$ y 51, de la ciudad de Barcelona que autorizaron y facilitaron el estudio. 
La disolución de la relación de pareja es cada vez más habitual en las sociedades industrializadas modernas (Andersson y Philipov 2002) y sus consecuencias afectan tanto a adultos como a menores (Amato 2000). Por analogía, desde la promulgación de la Ley del divorcio (Ley 30/ 1981), en España también la vida de muchas personas también se ha visto afectada. Según el Instituto Nacional de Estadística (2013) en 2012 se produjeron 110.764 disoluciones de las que 104.262 fueron divorcios, 6.369 separaciones, y 133 nulidades. El 34.1\% de los divorcios y el $27.9 \%$ de las separaciones fueron no consensuados.

En los expedientes de divorcio, donde se trate la guarda y custodia de menores debe predominar el principio del interés superior del menor (ISM) debido a la supremacía ratificada legalmente en la Convención de la Organización de Naciones Unidas (ONU) de 20 de noviembre de 1989, de los derechos del niño, y refrendada por España el 30 de noviembre de 1990 (Convención Derechos del Niño, 1990; ONU, 1989). El Derecho de familia interviene en esta para equilibrar las relaciones entre sus miembros y otorgar especial protección al menor por ser el que a priori presenta una posición más débil (de Torres, 2011).

En España, la función pericial está regulada en el Derecho Civil por la Ley de Enjuiciamiento Civil (LEC) y en el Derecho Penal en la Ley de Enjuiciamiento Criminal. Los peritos son profesionales con conocimientos especializados requeridos al proceso judicial para aportar aquéllos conocimientos técnicos que el Juez no tiene por qué poseer. Estos conocimientos son necesarios para la percepción y apreciación de hechos que no podrían captarse sin tal pericia. Así, el dictamen psicológico es un procedimiento legal a disposición del Juez para el ejercicio de su función judicial, tal como recoge el Art. 335 (LEC, 1/2000). Los dictámenes de evaluación de la custodia de menores (ECM) son elementos probatorios de las decisiones judiciales (Echeburúa, Muñoz y Loinaz, 2011) que inciden directamente en la vida de los menores, en la relación de éstos con sus progenitores y en su desarrollo evolutivo y psicosocial (Gardner, 1998; Muñoz, 2010). Cabe señalar que en España, alrededor del $80 \%$ de las demandas periciales psicológicas de los juzgados de familia corresponden a asuntos matrimoniales y de ellos una proporción variable pero no inferior a la mitad, son dictámenes de custodia (Arch y Jarne, 2010; Ramírez, 2003).

La American Psychological Association en sus 'Principios éticos de la Psicólogos y el Código de Conducta' indica unas recomendaciones sobre el comportamiento de los psicólogos destinada a facilitar el desarrollo sistemático y continuo del ejercicio profesional para sostener un elevado nivel ético y de calidad. Estas directrices no son normas obligatorias ni exhaustivas ya que no pueden ser aplicables a cada situación profesional. Por ello, la responsabilidad y el criterio final del informe recae en el psicólogo forense (APA, 2010).

La práctica forense de la evaluación de la custodia de menores (ECM) no ha estado exenta de controversias ampliamente descritas en la literatura sobre ausencia de métodos empíricos, uso inadecuado de test psicológicos, uso indebido o interpretación de los datos, falta de utilidad para el tribunal o actitudes sesgadas e imparcialidad (Bow y Quinnell, 2002, 2004).

En general, las principales cuestiones que la literatura ha puesto de manifiesto sobre la ECM se refieren a: 1) priorizar el ISM para minimizar el impacto del divorcio en el menor (de Torres, 2011); 2) necesidad de evaluar a todos los miembros del conjunto familiar (Molina, Arch y Jarne, 2012; Del Río, 2000; Gould y Martindale, 2007; Kuehnle, Greenberg y Gottlieb, 2004); 3) directrices (APA, 2013b; EFPA, 2005); 4) análisis de los contenidos del informe (APA, 1994; Bow y Quinnell, 2001); 5) preferencias sobre la custodia exclusiva o compartida (Alascio y Marín, 2007; Arch, 2010; de Torres, 2011); 6) uso de test y sus controversias, así como las diferencias entre evaluación clínica y evaluación forense (Echeburúa et al., 2011; Erard, 2007); 7) detección de la simulación (Arce y Fariña, 2005; Arce, Fariña y Carballal, 2009; Arce, Fariña y Seijo, 2005; Echeburúa et al., 2011; Manzanero y Muñoz, 2011); 8) conflictividad del divorcio (Austin y Drozd, 2013); 9) necesidad de modelos estandarizados de informe (Martindale et al., 2007); 10) ética y deontología del informe (Molina et al., 2012); y 11) actualización científica y formación especializada del psicólogo forense (APA, 2010a, 2013a, 2013b).

Tippins y Wittmann (2005) analizaron el 60\% de las evaluaciones de la custodia de menores presentadas en un tribunal estadounidense durante un período de dos años, conforme a las directrices de la APA (1994) y las diferencias basadas en la formación de los evaluadores. Hallaron una gran variedad de técnicas utilizadas e incoherencia entre las directrices de la APA y la práctica clínico-forense. Sus resultados propusieron la necesidad de criterios más normalizados de las ECM (Horvath, Logan y Walker, 2002). Se deberían incluir en los informes psicológicos advertencias sobre las limitaciones en la capacidad de pronosticar el tipo de custodia para una familia en concreto (Tippins y Wittmann, 2005). En la misma línea, jueces y fiscales, también expresaron la necesidad de mejoras en los informes de custodia de los hijos, focalizarlos en el menor, en las recomendaciones de la custodia y las pautas de contactos paterno-filiales, la valoración de criterios legales, y la adecuada conclusión de las evaluaciones (Bow y Quinnell, 2004).

En nuestro entorno, un estudio realizado en Andalucía (Aguilera y Zaldívar, 2003), consultaron a 42 jueces, que confirmaron la utilidad de las ECM para ayudarles en la toma de decisiones sobre los menores, respaldaron el papel decisivo que tiene el dictamen para valorar los aspectos centrales sobre la ECM como mejor alternativa de custodia en derecho de familia.

Otro estudio en Cataluña, (Domínguez, Gamero, González y Roca, 2009), analizó el impacto de los informes del "Servei d'Assessorament Tècnic en l'Àmbit de Família de Catalunya" (SATAF) en las resoluciones judiciales, confirmando sus autores, que los resultados de sus valoraciones tenían un destacado peso en las resoluciones judiciales. No obstante, en la práctica profesional en Cataluña, se detecta una controversia no resuelta en relación a la intervención; mientras los psicólogos privados 
(o peritos de parte) evalúan mayoritariamente a los menores, los técnicos del SATAF tienden a restringir sus evaluaciones según su guía de intervención técnica de menores donde razonan el principio de intervención mínima y el ISM como base para esta práctica y en su opinión, las ECM frecuentemente están contraindicadas optando por obtener la información secundariamente (SATAF, 2011). A pesar de estas recomendaciones, otros profesionales indican que no se podrán realizar afirmaciones en los dictámenes sobre personas que no se hayan explorado de forma directa y objetiva, por cuanto se debe evaluar directamente a los menores siempre que sea posible (Molina et al, 2012). Asimismo, recientemente, la Sala de lo Civil del Tribunal Supremo (2013), ordenó a la Audiencia Provincial de Orense a escuchar a los dos hijos de una pareja antes de decidir si concedía el régimen de custodia compartida o se la otorgaba a uno de los progenitores. El alto tribunal aludió a la Ley de Enjuiciamiento Civil, la Ley de Protección del Menor, al Convenio sobre los Derechos del Niño y la Carta Europea de Derechos Fundamentales para subrayar que, en los supuestos de que la edad y el desarrollo madurativo del niño/a permita suponer la suficiente capacidad de reflexión, y en todo caso, cuando sean mayores de 12 años habrán de ser escuchados en los procedimientos judiciales en los que se concluya sobre su guardia y custodia, sin que la parte pueda desistir a la propuesta probatoria, correspondiendo al juez de oficio decidirla en su caso (Tribunal Superior de Justicia, 2013).

Por otra parte, habitualmente se presentan informes psicológicos privados y ordenados por insaculación de los propios tribunales. Pese a su importancia, no conocemos más estudios publicados en nuestro país, con datos fiables sobre la estructura, metodología, y contenidos de la ECM comparando informes psicológicos privados e informes psicológicos de los equipos psicosociales, más allá de sugerencias teóricas sobre cómo efectuarlos que realizan las guías de recomendaciones y protocolos de organismos profesionales, colegios de psicólogos y comisiones deontológicas (APA, 1994, 2010a, 2010b, 2013a, 2013b; Bow, 2006; Chacón, García, Gómez, y Vázquez, 2009; De Torres, 2009, 2011).

Por ello, este trabajo pretende dar a conocer la situación de los informes periciales firmados por psicólogos privados y por profesionales del equipo psicosocial, basado en la evidencia, para contribuir así a mejorar los informes periciales bajo criterios de intervención reales, a la formación de los psicólogos forenses y comprender mejor la controversia suscitada por los peritos.

En base a ello siguiendo la 'Guía de buenas prácticas para la elaboración de informes psicológicos periciales sobre custodia y régimen de visita de menores' (Chacón, García, Gómez, y Vázquez, 2009) los objetivos del presente estudio han sido:

a) Conocer la estructura formal del informe pericial: su objetivo, metodología, resultados, discusión, conclusiones y recomendaciones: En este sentido, se entiende la estructuración del informe parcial (si hay ausencia de algún ele- mento de la estructura formal) o completa (si comprende todos los elementos).

b) Conocer similitudes o diferencias entre los informes de psicólogos privados (IP) e informes de profesionales del equipo psicosocial (IEPS).

En este estudio se consideraron las siguientes variables: si el informa se estructuró en apartados; qué tipo de informe fue: de competencia parental, de parentalidad + tipo de custodia, de cambio de régimen de visitas; qué cantidad de cuestiones planteó; la claridad de las cuestiones: si el lenguaje fue comprensible o utilizó tecnicismos innecesarios y si hubo influencia debido a tener o no formación específica forense; qué alegaciones aportó el informe: si presentó un trastorno psicológico activo, abuso sexual de menores, traslados, sustracción de menores, toxicomanía del cónyuge, evaluación del régimen de visitas, retirada de la potestad, modificación de medidas de oposición a la Dirección General de Atención a la Infancia y Adolescencia (DGAIA). Si entrevistó a los menores, a los progenitores, a todo el grupo familiar; si describió la relación del menor con la familia, con los progenitores, con los hermanos, con las nuevas parejas; si estudió el proceso judicial; los antecedentes de hecho; la disolución de la pareja; la coordinación telefónica en red con otros profesionales; si observó los menores en consulta, en medio natural; si diferenció, afirmó, infirió o interpretó las aportaciones del informante; si citó informes psicológicos anteriores; si comentó denuncias entre las partes; si reconoció las limitaciones del informe; si citó el art. 335 de la Ley de Enjuiciamiento Civil y la validez del informe; si respondió a las cuestiones planteadas; si incluyó apartado de conclusiones; observar si utilizó psicometría, si la relacionó con aspectos concretos del informe, si la utilizó con menores, si la utilizó con adultos; test utilizados con menores y con adultos; si incluyó las pruebas con los resultados; si dio puntuaciones estadísticas; lenguaje comprensible o muy técnico; si relacionó los resultados con los objetivos del informe; si detectó la simulación; descripción de las pruebas utilizadas en adultos y en menores; conocer si aportó conclusiones sobre la atribución de la custodia, sobre el régimen de visitas; si hizo recomendaciones en el informe sobre: psicoterapia individual, familiar, mediación, opciones pedagógicas y/o de recursos sociales.

De acuerdo a los objetivos del estudio, las hipótesis que exponemos, a fin de delimitar la presente investigación, fueron las siguientes:

1) Los autores de los informes serán mayoritariamente psicólogos profesionales de género femenino.

2) Se encontrarán más diferencias significativas en los informes emitidos por los IEPS, que en los informes emitidos por los psicólogos privados con formación específica forense (IPCF), y que en los psicólogos privados sin formación específica forense (IPSF), con relación una mayor estructuración, el tipo de informe, y las cuestiones planteadas. 
3) Se encontraran diferencias significativas entre los informes de los profesionales de los IEPS y los informes emitidos por los IP respecto a los contenidos y la metodología utilizados.

4) Se hallaran más diferencias significativas en el tipo de informe emitido por los IEPS que por los informes emitidos los IP, respecto a las garantías y limitación de los resultados, las afirmaciones del evaluado en las entrevistas y las recomendaciones utilizadas.

5) Los informes aportados por los IP utilizaran más pruebas psicométricas que los informes emitidos por los IEPS

6) Se pretende contrastar las diferencias observadas en los procedimientos utilizados en las evaluaciones de la custodia de menores entre el tipo de informe emitido por IP e informes emitidos por los IEPS, con el estudio de Bow y Quinnell (2002). Téngase en consideración que entre las diferencias del estudio de Bow y Quinnell (2002), y el presente estudio no es posible ni se pretende, la comparación exacta, por ser muestras de distinta procedencia.

\section{Participantes}

\section{Método}

Se analizaron 111 informes psicológicos periciales privados e informes periciales emitidos por los equipos psicosociales adscritos a los juzgados de familia y sentencias judiciales emitidas en primera instancia contenidas en los expedientes de divorcios contenciosos.

\section{Procedimiento}

Se extrajo una muestra preseleccionada de archivo que incluía informe pericial psicológico y sentencia emitida en primera instancia en el juzgado de familia. Se excluyeron otro tipo de informes firmados por otro tipo de profesional.

Se utilizaron 111 informes psicológicos, 45 privados y 66 del 'Equip d'Assessorament Tècnic en l'Àmbit de Família' (SATAF) incluidos en expedientes contenciosos, entre enero de 2007 y diciembre de 2013 en los Juzgados de Familia 15, 16, 17, 18, 19, 45, y 51 de Barcelona.

El estudio obtuvo resolución favorable del Comité de Ética de Investigación de la Universidad Ramón Llull (CERURL/2012/001). Para el estudio de los expedientes se solicitó autorización al Tribunal Superior de Justicia de Cataluña, que la trasladó con disposición favorable para su autorización a los Secretarios Judiciales de los juzgados de familia 15, 16, 17, 18, 19, 45, y 51 de la ciudad de Barcelona.

Se trata de un estudio ex post facto retrospectivo de tipo transversal que incluye algunas comparaciones entre grupos. Para la realización del estudio se confeccionó una plantilla post hoc elaborada por los autores que incluía información sobre el profesional signatario, especialización, tipo de informe solicitado, metodología del informe, contenidos del informe, garantías y limitación de los resultados, afirmaciones de los evaluados en las entrevistas, recomendaciones terapéuticas, pruebas psicométricas y los procedimientos utilizados en la evaluación de la custodia de menores.
El tratamiento estadístico tuvo finalidad descriptiva para lo cual se calcularon descriptores, frecuencias y análisis $\chi^{2}$ de las variables para averiguar si habían o no diferencias significativas $(p<.05 ; p<.01 ; p<.001)$ cuando se realizaron comparaciones entre grupos.

\section{Resultados}

Características demográficas de los profesionales autores de los informes de la muestra

El 100\% de los IP fueron emitidos por psicólogos. Por el contrario los informes IEPS fueron emitidos en un $74.2 \%$ por psicólogos y un $25.8 \%$ por trabajadores sociales. El $13.3 \%$ de los IP fueron emitidos por hombres y el $86.7 \%$ por mujeres. El $10.6 \%$ de los IEPS fueron emitidos por hombres y el $89.4 \%$ por mujeres.

El $66.7 \%$ de los IP fueron emitidos por un licenciado/graduado universitario, un $28.9 \%$ de los profesionales tenían el título de doctor, un $2.2 \%$ eran especialistas en psicología clínica y un $2.2 \%$ estaban especializados en psicología infantil. Un $64.4 \%$ tenía formación específica forense.

\section{Estructura del informe}

El $82 \%$ de todos los informes presentaron una estructura completa frente al $18 \%$ de los casos que fue parcial, pero con diferencias significativas según su procedencia. El informe completo de los IEPS presentó una frecuencia en mayor medida que los informes emitidos por IP $\left(\chi^{2}=3.832, p<.05\right)$, (tabla 1$)$.

Un $73 \%$ de los informes incluyeron evaluación de la parentalidad y tipo de custodia recomendado y el $26.1 \%$ restante solo parentalidad; los IEPS evaluaron en función de la orden del tribunal en mayor medida parentalidad que los IP que evaluaron a petición de una de las partes, pero en menor medida la recomendación de la custodia sin diferencias significativas (IEPS $65 \%$ vs. IP $84 \%$ ).

En general los informes psicológicos presentados ante los tribunales mostraron claridad en la exposición de las cuestiones a evaluar (IEPS 98.5\% vs IP 95.6\%) sin diferencias significativas. La estructuración en apartados de los IEPS mostró una diferencia significativa $\left(\chi^{2}=4.819, p<.05\right)$ en mayor medida respecto de los IP.

Globalmente en un 55\% de los informes se evaluó a los menores, siendo esta variable donde la diferencia entre ambos grupos fue mayor. Los IP entrevistaron a los hijos en un $88.9 \%$ de los casos en mayor medida que los IEPS en un $31.8 \%$, con diferencia estadísticamente significativa $\left(\chi^{2}=35.205, p<.001\right)$. No se hallaron diferencias significativas en cuanto a incluir entrevistas al padre (IEPS $89.4 \%$ vs IP $75 \%$ ), ni a toda la unidad familiar: padre, madre, hermanos y nuevas parejas (IEPS $28.8 \%$ vs $22.7 \%$ ). En cuanto a entrevistar a la madre (IEPS 93.9\% en mayor medida que IP 55.6\%) se hallaron diferencias significativas $\left(\chi^{2}=24.184, p<.001\right)$.

Los IEPS mostraron una frecuencia en mayor medida que los IP al describir la relación del menor con los miembros de la familia, con diferencias significativas $\left(\chi^{2}=4.247, p<.05\right)$; al 
describir la relación con el padre $\left(\chi^{2}=10.509, p<.01\right)$; con la madre $\left(\chi^{2}=9.603, p<.01\right)$; pero, sin diferencias significativas al describir la relación con los hermanos y la relación con las nuevas parejas.

Tabla 1

Estructura y Metodología del Informe.

\begin{tabular}{|c|c|c|c|}
\hline Estructura del Informe & Informes \% total & IP (\%) & IEPS (\%) \\
\hline \multicolumn{4}{|l|}{ Estructura del informe } \\
\hline Parcial & 18.0 & 26.7 & 12.1 \\
\hline Completo & 82.0 & 73.3 & $87.9^{*}$ \\
\hline \multicolumn{4}{|l|}{ Alegaciones del Informe } \\
\hline Trastorno psicológico activo & 13.0 & 15.4 & 12.5 \\
\hline Abuso sexual de menores & 4.3 & 15.4 & 1.8 \\
\hline Traslados & 7.2 & 23.1 & 3.6 \\
\hline Sustracción menores & 1.4 & 1.4 & - \\
\hline Toxicomanía del cónyuge & 7.2 & - & 8.9 \\
\hline Evaluación régimen visitas & 58.0 & 30.8 & 64.3 \\
\hline Retirada de la potestad & 7.1 & - & 7.1 \\
\hline \multicolumn{4}{|l|}{ Modificación de medidas de } \\
\hline oposición a la DGAIA & 1.4 & 7.7 & - \\
\hline \multicolumn{4}{|l|}{ Tipo de Informe } \\
\hline Parentalidad & 26.1 & 15.6 & 33.3 \\
\hline Parentalidad + tipo de Custodia & 73.0 & 84.4 & 65.2 \\
\hline Cambio de régimen de visitas & .9 & - & 1.5 \\
\hline \multicolumn{4}{|l|}{ Cuantas cuestiones plantea } \\
\hline Una & 27.9 & 31.1 & 25.8 \\
\hline Dos & 45.0 & 48.9 & 42.4 \\
\hline Tres o más & 27.0 & 20.0 & 31.8 \\
\hline \multicolumn{4}{|l|}{ Claridad de las cuestiones } \\
\hline $\mathrm{Si}$ & 97.3 & 95.6 & 98.5 \\
\hline No & 2.7 & 4.4 & 1.5 \\
\hline \multicolumn{4}{|l|}{ Estructuración en apartados } \\
\hline $\mathrm{Si}$ & 94,6 & 88.8 & $98.5^{*}$ \\
\hline No & 5,4 & 11.1 & 1.5 \\
\hline \multicolumn{4}{|l|}{ Entrevistó hijos/as } \\
\hline $\mathrm{Si}$ & 55.0 & $88.9 * * *$ & 31.8 \\
\hline No & 45.0 & 11.1 & 68.2 \\
\hline \multicolumn{4}{|l|}{ Entrevistó al padre } \\
\hline $\mathrm{Si}$ & 83.6 & 75.0 & 89.4 \\
\hline No & 14.5 & 20.5 & 10.6 \\
\hline Pero fue citado & 1.8 & 4.5 & - \\
\hline \multicolumn{4}{|l|}{ Entrevistó a la madre } \\
\hline $\mathrm{Si}$ & 78.4 & 55.6 & $93.9 * * *$ \\
\hline No & 16.2 & 31.1 & 6.1 \\
\hline Pero fue citada & 5.4 & 13.3 & - \\
\hline \multicolumn{4}{|l|}{ Entrevistó todo el grupo } \\
\hline $\mathrm{Si}$ & 26.1 & 22.7 & 28.8 \\
\hline No & 73.9 & 77.8 & 71.2 \\
\hline \multicolumn{4}{|l|}{$\begin{array}{l}\text { Describe la relación del } \\
\text { menor con la familia }\end{array}$} \\
\hline $\mathrm{Si}$ & 92.8 & 86.7 & $97.0 *$ \\
\hline No & 7.2 & 13.3 & 3.0 \\
\hline \multicolumn{4}{|l|}{$\begin{array}{l}\text { Describe la relación del } \\
\text { menor con el padre }\end{array}$} \\
\hline $\mathrm{Si}$ & 89.1 & 77.3 & $97.0 * *$ \\
\hline No & 10.9 & 22.7 & 3.0 \\
\hline \multicolumn{4}{|l|}{$\begin{array}{l}\text { Describe la relación del } \\
\text { menor con la madre }\end{array}$} \\
\hline $\mathrm{Si}$ & 87.4 & 75.6 & $95.5^{* *}$ \\
\hline No & 12.6 & 24.4 & 4.5 \\
\hline \multicolumn{4}{|l|}{$\begin{array}{l}\text { Describe la relación del } \\
\text { menor con los hermanos }\end{array}$} \\
\hline $\mathrm{Si}$ & 49.5 & 58.3 & 43.6 \\
\hline No & 50.5 & 41.7 & 56.4 \\
\hline \multicolumn{4}{|l|}{$\begin{array}{l}\text { Describe la relación del } \\
\text { menor con las nuevas parejas }\end{array}$} \\
\hline $\mathrm{Si}$ & 17.0 & 22.5 & 13.6 \\
\hline No & 83.0 & 77.5 & 86.4 \\
\hline
\end{tabular}

Nota: IP: Informe Psicólogo privado. IEPS: Informe equipo psicosocial. DGAIA: Dirección general de atención a la infancia y adolescencia. $* p<.05 ; * * p<.01 ; * * * p<001$

\section{Nivel de formación específica forense}

En los informes IP se analizó si había diferencias relativas a la formación específica forense con los que no describieron poseer formación específica forense (tabla 2). Globalmente los informes de los IP fueron estructurados en apartados, pero al relacionar la formación específica forense en los IPCF, se hallaron diferencias significativas $\left(\chi^{2}=10.195, p<.01\right)$ en mayor medida respecto a los IPSF, dato que ratifica la necesidad de la formación específica forense.

Respecto al estudio del proceso judicial en su conjunto alcanzó un 73.3\%, pero los IPCF obtuvieron diferencias significativas en mayor medida respecto de los IPSF al comparar ambos grupos $\left(\chi^{2}=11.111, p<.01\right)$.

Al relacionar la explicación de los antecedentes de hecho globalmente se obtuvo un $82 \%$, pero los IPCF obtuvieron diferencias significativas $\left(\chi^{2}=11.457, p<.01\right)$ en mayor medida respecto de los IPSF, al comparar ambos grupos.

Al analizar la disolución de la pareja globalmente obtuvo un $63.6 \%$, pero al comparar los grupos, los IPCF, mostraron diferencias significativas $\left(\chi^{2}=7.422, p<.01\right)$ en mayor medida respecto de los IPSF.

La coordinación en red con otros profesionales fue sólo de un $17.8 \%$, sin diferencias significativas, aunque fue mayor en el IPCF (24.1\%) que en el IPSF (6.3\%). Es importante destacar la necesidad de incrementar la coordinación en red, para una contratación adecuada de las ECM.

Tabla 2

Nivel de formación específica forense (Informes privados).

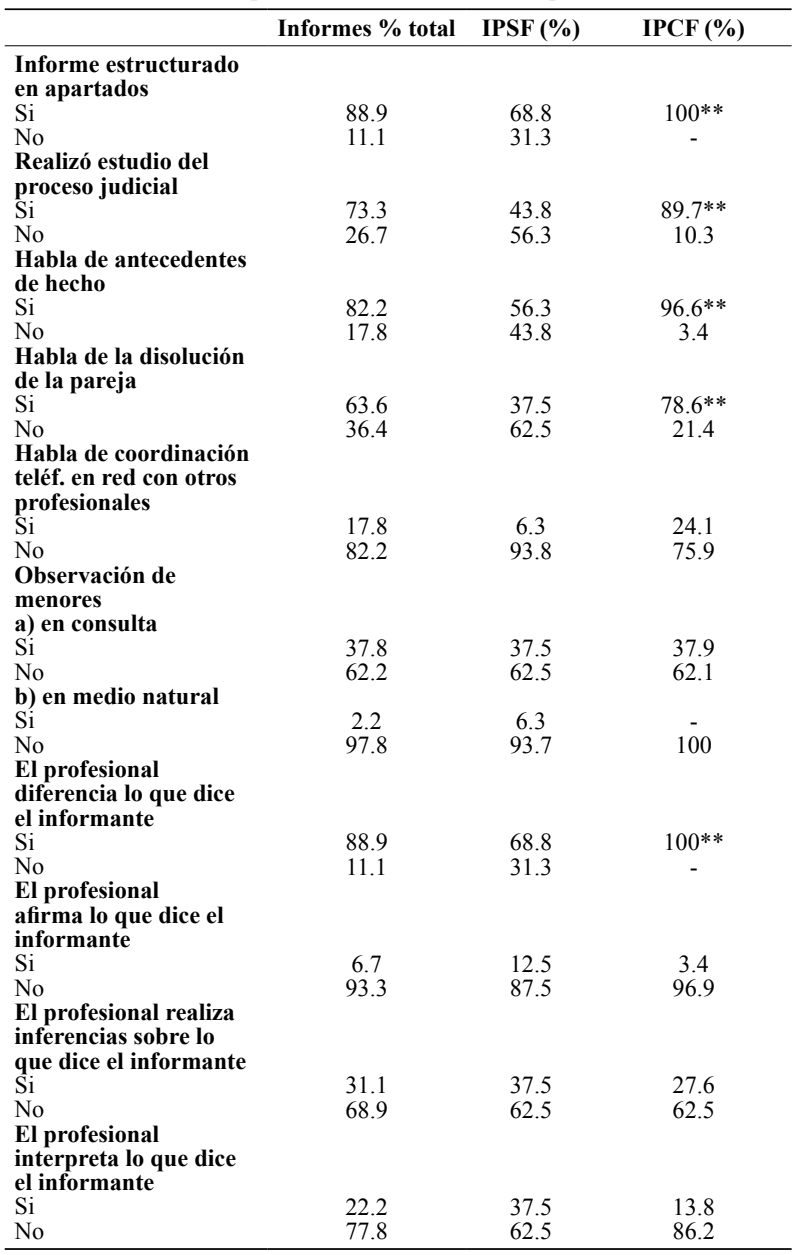

Nota: IPSF: Informe psicólogo sin formación forense, IPCF: Informe psicólogo con formación forense. ${ }^{* *} p<.01$ 
En relación a la observación de menores en consulta, en medio natural (domicilio), no se observaron diferencias significativas. Por otro lado, ambos grupos, diferenciaron en un $88.9 \%$ lo que dice el informante, pero los IPCF mostraron diferencias significativas $\left(\chi^{2}=10.195, p<.01\right)$ en mayor medida respecto de los IPSF.

Sólo un $22.2 \%$ interpretó lo que dijo el informante. Pese a no haber diferencias significativas entre grupos, las interpretaciones del IPCF fueron menores, que las del IPSF, otro tanto sucedió con las inferencias: IPCF $27.6 \%$ vs IPSF $37.5 \%$, y respecto de la realización de afirmaciones del profesional respecto de lo que dice el informante: IPCF $3.4 \%$ vs IPSF $12.5 \%$.

Tipo de informe y recomendaciones sobre Guarda y Custodia y tipo de régimen de visitas

Al comparar tipo de informe y recomendaciones sobre la guarda y custodia y régimen de contactos con el progenitor no custodio, según su procedencia: IPCF, IPSF y IEPS, obtuvimos los siguientes resultados (tabla 3).

El tipo de informe completo obtuvo diferencias significativas $\left(\chi^{2}=8.644, p<.05\right)$ siendo un $87.7 \%$ en los IEPS, un $88.3 \%$ en los IPCF, pero un $56.3 \%$ en los IPSF. Lo que puede sugerir que la utilización de protocolos facilita una mejor estructuración de los dictámenes.

En relación a entrevistar a toda la unidad familiar, aunque no tuvieron diferencias significativas, se resalta que sólo un $27.7 \%$ de los IEPS, un $26.7 \%$ de los IPCF, y un $18,8 \%$ de los IPSF completaron las entrevistas a toda la unidad familiar. Por contra, en relación al dato anterior sorprende que aportaran recomendaciones sobre la guarda y custodia un $62.2 \%$ de los IEPS, un $60 \%$ de los IPCF, y un 70\% de los IPSF, aunque, sin diferencias significativas.

$\mathrm{Al}$ aportar recomendaciones sobre el régimen de visitas con el progenitor no custodio, los IPCF mostraron una mayor tasa respecto de los IPSF y de los IEPS, con una diferencia significativa $\left(\chi^{2}=12.787, p<.01\right)$, aspecto que puede estar relacionado con procedencia de la demanda judicial al IEPS, o por uno de los litigantes al IPCF o IPSF.

Tabla 3

Tipo de informe y recomendaciones sobre Guarda y Custodia y Régimen de Visitas.

\begin{tabular}{lccc}
\hline $\begin{array}{l}\text { Estructura informe/ } \\
\text { Entrevista todo el grupo/ } \\
\text { Aporta consideración GC } \\
\text { y RC }\end{array}$ & IPSF (\%) & IPCF (\%) & IEPS (\%) \\
\hline $\begin{array}{l}\text { Tipo de informe } \\
\text { Parcial }\end{array}$ & 43.8 & 16.7 & \\
$\begin{array}{l}\text { Completo } \\
\text { Entrevistó todo el grupo }\end{array}$ & 56.3 & $88.3^{*}$ & 87.3 \\
SI & 18.8 & 26.7 & 27.7 \\
NO & 81.3 & 73.3 & 72.3 \\
$\begin{array}{l}\text { Aporta recomendaciones } \\
\text { Sobre Guarda y Custodia }\end{array}$ & & & \\
SI & 70 & 60 & 62.2 \\
NO & 30 & 40 & 37.8 \\
Aporta recomendaciones & & & \\
Sobre Régimen de visitas & 55.2 & $79.7^{* *}$ & 67.0 \\
SI & 44.8 & 20.3 & 33.0 \\
\hline NO &
\end{tabular}

Nota: IPSF: Informe Psicólogo sin formación forense, IPCF: Informe psicólogo con formación forense, IEPS: Informe equipo psicosocial. $* p<.05 ; * * p<.01$

\section{Contenidos de la evaluación descritos en el informe pericial}

Una cuarta parte del total de los informes reconoció las limitaciones de los informes, pero, al comparar los IP y IEPS, no se hallaron diferencias significativas. Sin embargo, respecto a citar el art. 335 de la LEC y la validez temporal del informe, los IP obtuvieron diferencias significativas $\left(\chi^{2}=52.134, p<\right.$ .001) en mayor medida que los IEPS.

En conjunto casi la mitad de los informes incluyeron la psicometría, y la relacionaron con los aspectos concretos de la pericial. Los IP obtuvieron respecto de los IEPS una mayor tasa al incluir la psicometría $\left(\chi^{2}=55.499, p<.001\right)$, y al relacionarla con los aspectos concretos de la pericial $\left(\chi^{2}=42.458, p<.001\right)$, con una diferencia significativa.

Globalmente, más de tres cuartas partes de los profesionales realizaron un estudio sobre los datos del expediente judicial. Aunque, los IEPS mostraron diferencias significativas $\left(\chi^{2}=\right.$ $19.733, p<.001$ ) en mayor medida respecto de los IP.

Más del $80 \%$ de los firmantes de todos los informes comentaron los antecedentes de hecho y de la disolución de la relación de la pareja. Aunque, los IEPS, en los antecedentes de hecho presentaron diferencias significativas $\left(\chi^{2}=7.099, p<.01\right)$, en mayor medida con respecto a los IP, y también en la disolución de la relación de la pareja $\left(\chi^{2}=12.273, p<.01\right)$.

Casi un $30 \%$ del total, citaron informes psicológicos anteriores pero de éstos, los IP lograron diferencias significativas en mayor medida respecto de los IEPS $\left(\chi^{2}=7.683, p<.01\right)$. Sin embargo, no se encontraron diferencias significativas al comentar las denuncias entre las partes.

Al recabar información mediante la coordinación con otros profesionales relacionados con el menor: casi la mitad del total solicitaron información a profesores, pero, los IEPS consiguieron diferencia significativa $\left(\chi^{2}=4.262, p<.05\right)$ en mayor medida respecto de los IP; respecto a la coordinación con médicos o pediatras no hubo diferencias significativas; en la coordinación telefónica con profesionales, los IEPS obtuvieron diferencia significativa en mayor medida que los IP $\left(\chi^{2}=\right.$ $30.561, p<.001$ ), la coordinación presencial con otros profesionales no hubo diferencias significativas entre ambos grupos. En la coordinación con el 'punto de encuentro supervisado', los IEPS, tuvieron diferencias significativas $\left(\chi^{2}=9.174, p<.01\right)$ en mayor medida con relación a los IP que no realizaron la coordinación con el punto de encuentro supervisado.

Los informes de ambos grupos estudiados no manifestaron realizar observación de menores en el medio natural (domicilio del menor). Casi un tercio del total de firmantes utilizaron la psicometría en adultos y en menores, pero los IP obtuvieron una diferencia significativa al usar la psicometría en menores $\left(\chi^{2}=\right.$ $32.964, p<.001)$, y en adultos $\left(\chi^{2}=39.284, p<.001\right)$ en mayor medida respecto de los IEPS.

El estudio de la relación entre la madre y los hijos, de forma global los informes realizados superaron la tasa del 80\%, sin embargo, los IEPS alcanzaron diferencias significativas $\left(\chi^{2}=\right.$ $19.394, p<.001$ ) en mayor medida respecto de los IP, lo cual resulta factible con el rechazo del otro progenitor en litigio a ser 
evaluados por un IP de la otra parte, observado con frecuencia en la práctica cotidiana; en la misma dirección la relación entre el padre y los menores, los IEPS mostraron diferencias significativas $\left(\chi^{2}=8.041, p<.01\right)$ en mayor medida respecto de los IP; el análisis de la relación entre los progenitores, los IEPS consiguieron diferencias significativas $\left(\chi^{2}=12.160, p<.01\right)$ en mayor medida respecto de los IP. No se hallaron diferencias significativas respecto a hablar de las nuevas parejas (tabla 4), sin embargo, resulta necesario un análisis de las relaciones del menor con la nueva pareja, cuando se constituye una nueva familia.

Garantías, limitación de los resultados, valoración del profesional sobre las afirmaciones del informante en las entrevistas y recomendaciones del informe psicológico

Globalmente, el análisis de ambos grupos concordó con los datos estudiados, sin diferencias significativas (tabla 5), sin embargo, respecto a las referencias sobre la comprobación de la veracidad del testimonio, sólo la realizaron un $14.4 \%$, aunque, los IEPS obtuvieron, diferencias significativas $\left(\chi^{2}=6.098, p<\right.$ $.05)$ en mayor medida respecto de los IP. En la mitad del total de informes, se contrastó la información aportada por terceros. Los IEPS lograron, diferencias significativas $\left(\chi^{2}=17.382, p<\right.$ .001) con más del doble de casos, respecto a los IP.

En general, una quinta parte de los informes periciales refirieron las limitaciones del estudio. Aún sin mostrar diferencias significativas, los IP tuvieron una mayor medida de reconocimiento de las limitaciones que los IEPS (26.7\% vs. $19.7 \%)$. Aspecto que contrasta, con el hecho de que todos los informes respondieron a las cuestiones planteadas con una tasa superior al $90 \%$, sin diferencias significativas. Es importante referir las limitaciones del dictamen, en relación a facilitar la tarea judicial.

Respecto a incluir un apartado de conclusiones, todos los informes mostraron una tasa cercana al $90 \%$, sin diferencias significativas. En general, alrededor del $60 \%$ de todos los informes aportaron conclusiones sobre la atribución de la custodia, sin diferencias significativas. En total, un $67 \%$ de todos los informes aportaron conclusiones sobre la atribución del régimen de visitas, aunque los IEPS obtuvieron, diferencias significativas $\left(\chi^{2}=12.355, p<.001\right)$ en mayor medida respecto de los IP.

Al diferenciar las afirmaciones del informante entrevistado, globalmente todos los informes alcanzaron aproximadamente un $90 \%$, aunque los IEPS, obtuvieron diferencias significativas $\left(\chi^{2}=4.819, p<.05\right)$ en mayor medida respecto de los IP. Prácticamente no se realizaron afirmaciones de lo que decían los informantes, sin diferencias significativas. Sin embargo, si se realizaron inferencias en un $13.5 \%$ del total de los informes, pero los IEPS obtuvieron, diferencias significativas $\left(\chi^{2}=\right.$ $20.053, p<.001)$ en mayor medida respecto de los IP. Sólo un $10.8 \%$ del total, realizó interpretaciones sobre lo que dijeron los informantes, si bien los IEPS, mostraron diferencias signi-
Tabla 4

Contenidos de la evaluación descritos en el informe pericial.

\begin{tabular}{|c|c|c|c|}
\hline Metodología & $\begin{array}{c}\text { Informes \% } \\
\text { total }\end{array}$ & IP (\%) & IEPS $(\%)$ \\
\hline \multicolumn{4}{|l|}{ Reconoce limitaciones del informe } \\
\hline $\mathrm{Si}$ & 25.2 & 28.9 & 22.7 \\
\hline No & 74.8 & 71.1 & 77.3 \\
\hline \multicolumn{4}{|l|}{$\begin{array}{l}\text { Cita el Art. } 335 \text { LEC y validez del } \\
\text { informe }\end{array}$} \\
\hline $\mathrm{Si}$ & 30.6 & $68.9 * * *$ & 4.5 \\
\hline No & 69.4 & 31.1 & 95.5 \\
\hline \multicolumn{4}{|l|}{ Utiliza psicometría } \\
\hline $\mathrm{Si}$ & 44.1 & $86.7 * * *$ & 15.2 \\
\hline No & 55.9 & 13.3 & 84.8 \\
\hline \multicolumn{4}{|l|}{$\begin{array}{l}\text { Relaciona psicometría con aspectos } \\
\text { concretos }\end{array}$} \\
\hline $\mathrm{Si}$ & 47.7 & $86.0 * * *$ & 21.9 \\
\hline No & 52.3 & 14.0 & 78.1 \\
\hline \multicolumn{4}{|l|}{ Realizó estudio del proceso judicial } \\
\hline $\mathrm{Si}$ & 89.2 & 73.3 & $100 * * *$ \\
\hline No & 10.8 & 26.7 & - \\
\hline \multicolumn{4}{|l|}{ Habla de antecedentes de hecho } \\
\hline $\mathrm{Si}$ & 91.9 & 82.2 & $97.0 * *$ \\
\hline No & 9.0 & 17.8 & 3.0 \\
\hline \multicolumn{4}{|l|}{$\begin{array}{l}\text { Habla de la disolución matrimonial } \\
\text { o de la pareja }\end{array}$} \\
\hline $\mathrm{Si}$ & 80.0 & 63.6 & $90.9 * *$ \\
\hline No & 20.0 & 36.4 & 9.1 \\
\hline \multicolumn{4}{|l|}{$\begin{array}{l}\text { Cita informes psicológicos } \\
\text { anteriores }\end{array}$} \\
\hline $\mathrm{Si}$ & 27.9 & $42.2 * *$ & 18.2 \\
\hline No & 72.1 & 57.8 & 81.8 \\
\hline \multicolumn{4}{|l|}{ Comenta denuncias entre las partes } \\
\hline $\mathrm{Si}$ & 22.5 & 20.0 & 24.2 \\
\hline No & 77.5 & 80.0 & 75.8 \\
\hline \multicolumn{4}{|l|}{$\begin{array}{l}\text { Habla de coordinación con los } \\
\text { profesores }\end{array}$} \\
\hline $\mathrm{Si}$ & 40.5 & 28.9 & $48.5^{*}$ \\
\hline No & 59.5 & 71.1 & 51.5 \\
\hline \multicolumn{4}{|l|}{$\begin{array}{l}\text { Habla de coordinación con médicos } \\
\text { o pediatras }\end{array}$} \\
\hline $\mathrm{Si}$ & 31.5 & 31.1 & 31.8 \\
\hline No & 68.5 & 68.9 & 68.2 \\
\hline \multicolumn{4}{|l|}{$\begin{array}{l}\text { Habla de coordinación tel. en red } \\
\text { con profesionales }\end{array}$} \\
\hline $\mathrm{Si}$ & 49.5 & 17.8 & $71.2 * * *$ \\
\hline No & 50.5 & 82.2 & 28.8 \\
\hline \multicolumn{4}{|l|}{$\begin{array}{l}\text { Comenta si hizo coordinación } \\
\text { presencial con otros profesionales }\end{array}$} \\
\hline $\mathrm{Si}$ & 1.8 & 2.2 & 1.5 \\
\hline No & 98.2 & 97.8 & 98.5 \\
\hline \multicolumn{4}{|l|}{$\begin{array}{l}\text { Observación de menores en el } \\
\text { medio natural }\end{array}$} \\
\hline $\mathrm{Si}$ & .9 & 2.2 & - \\
\hline No & 99.1 & 97.8 & 100.0 \\
\hline \multirow{2}{*}{\multicolumn{4}{|c|}{ Coordinación con el Punt de }} \\
\hline & & & \\
\hline $\mathrm{Si}$ & 10.8 & - & $18.2 * *$ \\
\hline No & 89.2 & 100.0 & 81.8 \\
\hline $\begin{array}{l}\text { Emplea psicometría con los } \\
\text { menores }\end{array}$ & & & \\
\hline $\mathrm{Si}$ & 33.3 & $64.4 * * *$ & 12.1 \\
\hline No & 66.6 & 35.6 & 87.9 \\
\hline Emplea psicometría con los adultos & & & \\
\hline $\mathrm{Si}$ & 26.1 & $57.8 * * *$ & 4.5 \\
\hline No & 73.9 & 42.2 & 95.5 \\
\hline $\begin{array}{l}\text { El profesional explica la relación } \\
\text { entre la madre e hijos }\end{array}$ & & & \\
\hline $\mathrm{Si}$ & 80.2 & 60.0 & $93.9 * * *$ \\
\hline No & 19.8 & 40.0 & 6.1 \\
\hline $\begin{array}{l}\text { El profesional explica la relación } \\
\text { entre el padre e hijos }\end{array}$ & & & \\
\hline $\mathrm{Si}$ & 86.4 & 75.0 & $93.9 * *$ \\
\hline No & 13.6 & 25.0 & 6.1 \\
\hline $\begin{array}{l}\text { El profesional explica la relación } \\
\text { entre los progenitores }\end{array}$ & & & \\
\hline $\mathrm{Si}$ & 78.2 & 61.4 & $89.4 * *$ \\
\hline No & 21.8 & 38.6 & 10.6 \\
\hline $\begin{array}{l}\text { El profesional explica la relación } \\
\text { con las nuevas parejas }\end{array}$ & & & \\
\hline $\mathrm{Si}$ & 13.5 & 15.6 & 12.1 \\
\hline No & 86.5 & 84.4 & 87.9 \\
\hline
\end{tabular}

Nota: IP: Informe Psicólogo privado. IEPS: Informe equipo psicosocial.

$p<* .05 ; p<* * .01 ; p<* * * .001$ 
Tabla 5

Garantías y limitación de los resultados, valoración del profesional sobre las afirmaciones del informante en las entrevistas y recomendaciones del informe psicológico.

\begin{tabular}{|c|c|c|c|}
\hline $\begin{array}{l}\text { Valoraciones del } \\
\text { profesional }\end{array}$ & $\begin{array}{c}\text { Informes \% } \\
\text { total }\end{array}$ & IP (\%) & IEPS (\%) \\
\hline \multicolumn{4}{|l|}{$\begin{array}{l}\text { El análisis concuerda } \\
\text { con los datos } \\
\text { estudiados }\end{array}$} \\
\hline $\mathrm{Si}$ & 89.0 & 95.6 & 84.4 \\
\hline No & 11.0 & 4.4 & 15.6 \\
\hline \multicolumn{4}{|l|}{$\begin{array}{l}\text { Hace referencia a } \\
\text { la veracidad del } \\
\text { testimonio }\end{array}$} \\
\hline $\mathrm{Si}$ & 14.4 & 4.4 & $21.2 *$ \\
\hline No & 85.6 & 95.6 & 78.8 \\
\hline \multicolumn{4}{|l|}{$\begin{array}{l}\text { Contrasta con } \\
\text { información aportada } \\
\text { por terceros }\end{array}$} \\
\hline $\mathrm{Si}$ & 55.0 & 31.1 & $71.2 * * *$ \\
\hline No & 45.0 & 68.9 & 28.8 \\
\hline \multicolumn{4}{|l|}{$\begin{array}{l}\text { Refiere limitaciones } \\
\text { del estudio }\end{array}$} \\
\hline $\mathrm{Si}$ & 22.5 & 26.7 & 19.7 \\
\hline No & 77.5 & 73.3 & 80.3 \\
\hline \multicolumn{4}{|l|}{$\begin{array}{l}\text { Responde a las } \\
\text { cuestiones planteadas }\end{array}$} \\
\hline $\mathrm{Si}$ & 95.5 & 93.3 & 97.0 \\
\hline No & 4.5 & 6.7 & 3.0 \\
\hline \multicolumn{4}{|l|}{$\begin{array}{l}\text { Incluye apartado de } \\
\text { conclusiones }\end{array}$} \\
\hline $\mathrm{Si}$ & 93.7 & 88.9 & 97.0 \\
\hline No & 6.3 & 11.1 & 3.0 \\
\hline \multicolumn{4}{|l|}{$\begin{array}{l}\text { Aporta conclusiones } \\
\text { sobre atribución de la } \\
\text { custodia }\end{array}$} \\
\hline $\mathrm{Si}$ & 62.2 & 64.4 & 60.6 \\
\hline No & 37.8 & 35.6 & 39.4 \\
\hline \multicolumn{4}{|l|}{$\begin{array}{l}\text { Aporta conclusiones } \\
\text { sobre atribución del } \\
\text { régimen de visitas }\end{array}$} \\
\hline $\mathrm{Si}^{\circ}$ & 67.0 & 47.7 & $80.0 * * *$ \\
\hline No & 33.0 & 52.3 & 20.0 \\
\hline \multicolumn{4}{|l|}{$\begin{array}{l}\text { Diferencia las } \\
\text { afirmaciones del } \\
\text { informante }\end{array}$} \\
\hline $\mathrm{Si}$ & 94.6 & 88.9 & $98.5^{*}$ \\
\hline No & 5.4 & 11.1 & 1.5 \\
\hline \multicolumn{4}{|l|}{$\begin{array}{l}\text { Afirma lo que dice el } \\
\text { informante }\end{array}$} \\
\hline $\mathrm{Si}$ & 2.7 & 6.7 & - \\
\hline No & 97.3 & 93.3 & 100 \\
\hline \multicolumn{4}{|l|}{$\begin{array}{l}\text { Infiere lo que dice el } \\
\text { informante }\end{array}$} \\
\hline $\mathrm{Si}$ & 13.5 & $31.1 * * *$ & 1.5 \\
\hline No & 97.3 & 68.9 & 98.5 \\
\hline \multicolumn{4}{|l|}{$\begin{array}{l}\text { Interpreta lo que dice } \\
\text { el informante }\end{array}$} \\
\hline $\mathrm{Si}$ & 10.8 & $22.2 * *$ & 3.0 \\
\hline No & 89.2 & 77.8 & 97.0 \\
\hline \multicolumn{4}{|l|}{$\begin{array}{l}\text { Recomendaciones } \\
\text { del informe }\end{array}$} \\
\hline $\begin{array}{l}\text { No hace } \\
\text { recomendaciones }\end{array}$ & 63.1 & 64.4 & 62.1 \\
\hline \multicolumn{4}{|l|}{$\begin{array}{l}\text { Psicoterapia } \\
\text { individual para ambos }\end{array}$} \\
\hline padres & 4.5 & 4.4 & 4.5 \\
\hline Psicoterapia familiar & 5.4 & 2.2 & 7.6 \\
\hline \multicolumn{4}{|l|}{ Psicoterapia } \\
\hline $\begin{array}{l}\text { individual para la } \\
\text { madre }\end{array}$ & 5.3 & 11.1 & 18.2 \\
\hline \multicolumn{4}{|l|}{$\begin{array}{l}\text { Psicoterapia } \\
\text { individual para el }\end{array}$} \\
\hline padre & 3.6 & 2.2 & 4.5 \\
\hline \multicolumn{4}{|l|}{$\begin{array}{l}\text { Psicoterapia } \\
\text { individual para los }\end{array}$} \\
\hline hijos/as & 6.3 & 11.1 & 3.0 \\
\hline Mediación familiar & 6.3 & 4.4 & 7.6 \\
\hline Opciones & & & \\
\hline psicopedagógicas & 11.7 & 4.4 & - \\
\hline $\begin{array}{l}\text { Opciones de recursos } \\
\text { sociales }\end{array}$ & 10.4 & 6.7 & 15.2 \\
\hline
\end{tabular}

Nota: IP: Informe Psicólogo privado. IEPS: Informe equipo psicosocial.

$* p<.05 ; * * p<.01 ; * * * p<.001$ ficativas $\left(\chi^{2}=10.221, p<.01\right)$ en mayor medida respecto de los IP. Alrededor del $60 \%$ de todos los informes no realizaron recomendaciones específicas, pero aun así, la psicoterapia familiar (15.3) fue la más recomendada.

\section{Pruebas psicométricas utilizadas en los informes periciales}

De forma global alrededor de un tercio de los dictámenes incluyeron pruebas psicométricas para los adultos y para los menores evaluados (tabla 6). Se observó que los IP, mostraron diferencias significativas $\left(\chi^{2}=39.284, p<.001\right)$, en la administración de pruebas a adultos, $\mathrm{y}\left(\chi^{2}=24.162, p<.001\right)$, a los menores, al incluir las pruebas con los resultados $\left(\chi^{2}=16.370\right.$, $p<.001)$, al dar puntuaciones estadísticas $\left(\chi^{2}=16.370, p<\right.$ $.001)$, al relacionar los resultados de las pruebas psicométricas con los objetivos del informe $\left(\chi^{2}=46.425, p<.001\right)$, en mayor medida respecto de los IEPS.

En ambos grupos, el uso del leguaje fue comprensible sin utilizar jerga técnica, sin diferencias significativas. Por otro lado, prácticamente la totalidad de los informes de ambos grupos no expresaron si detectaron la simulación, sin diferencias significativas.

Respecto al uso de pruebas psicométricas, encontramos una amplia gama de pruebas utilizadas, con una mayoritaria preferencia del test 'CUIDA' para adultos, seguido del 'MMPI', y una predilección de los test tipo proyectivos en los menores seguida por el test 'TAMAI'.

\section{Procedimientos utilizados en las evaluaciones de la custodia de menores}

Entre los procedimientos utilizados en las evaluaciones de la custodia de menores (tabla 7), se muestran los resultados obtenidos del estudio de Bow y Quinnell (2002), y los resultados del presente estudio, advirtiendo al lector, la salvedad de que no se pueden comparar directamente por proceder de muestras distintas, y considerando la cautela de que la legislación estadounidense es muy estricta con sus operadores jurídicos, y que difiere de nuestro sistema legislativo, pero entendemos que puede ser de utilidad como pauta de referencia.

La primeras diferencias importantes con el estudio de Bow y Quinnell (2002), es que entrevistaron la totalidad de ambos progenitores, y a casi la totalidad de los menores, dato que muestra diferencias sustanciales con nuestro estudio. En su estudio, se manifiesta una tasa mayor del doble de observaciones del menor en consulta que los informes de nuestro estudio. Respecto de los test objetivos de personalidad el estudio de Bow y Quinnell (2002), prácticamente dobla el porcentaje en todas las pruebas, excepto si consideramos los inventarios de parentalidad (que en nuestro estudio hemos considerado el test “cuida"). Inversamente, en el estudio de Bow y Quinnell (2002), se puede observar que se utilizan en menor frecuencia los test en los menores, frente a los IP que los usan en una elevada proporción. 
Tabla 6

Pruebas psicométricas utilizadas en los informes periciales.

\begin{tabular}{|c|c|c|c|}
\hline & Informes \% total & IP (\%) & IEPS (\%) \\
\hline \multicolumn{4}{|l|}{ Test utilizados con los adultos } \\
\hline 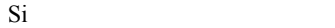 & 26.1 & $57.8^{* * *}$ & 4.5 \\
\hline No & 73.9 & 42.2 & 95.5 \\
\hline \multicolumn{4}{|l|}{ Test utilizados con los menores } \\
\hline $\mathrm{Si}$ & 33.3 & $55.6^{* * * *}$ & 12.1 \\
\hline No & 67.7 & 44.4 & 87.9 \\
\hline \multicolumn{4}{|l|}{$\begin{array}{l}\text { Incluye pruebas con los } \\
\text { resultados }\end{array}$} \\
\hline $\mathrm{Si}$ & 11.7 & $26.7^{* * *}$ & 1.5 \\
\hline No & 88.3 & 73.3 & 98.5 \\
\hline \multicolumn{4}{|l|}{ Da puntuaciones estadísticas } \\
\hline $\mathrm{Si}$ & 11.7 & $26.7^{* * *}$ & 1.5 \\
\hline \multirow{2}{*}{\multicolumn{4}{|c|}{ Usa lenguaje comprensible }} \\
\hline & & & \\
\hline $\mathrm{Si}$ & 99.1 & 97.8 & 100 \\
\hline No & .9 & 2.2 & - \\
\hline \multicolumn{4}{|l|}{ Usa lenguaje muy técnico } \\
\hline $\begin{array}{l}\mathrm{Si} \\
\text { No }\end{array}$ & .9 & 2.2 & \\
\hline & 99.1 & 97.8 & 100 \\
\hline \multicolumn{4}{|l|}{$\begin{array}{l}\text { Relaciona los resultados con los } \\
\text { objetivos del informe }\end{array}$} \\
\hline $\mathrm{Si}^{\circ}$ & 44.5 & $84.1^{* * * *}$ & 18.2 \\
\hline \multirow{2}{*}{\multicolumn{4}{|c|}{ Explica si detecta la simulación }} \\
\hline & & & \\
\hline $\mathrm{Si}^{2}$ & .9 & 2.2 & - \\
\hline No & 99.0 & 97.8 & 100 \\
\hline \multicolumn{4}{|l|}{ Pruebas utilizadas con adultos } \\
\hline $\begin{array}{l}\text { MMPI } \\
\text { NEO-PI-R }\end{array}$ & 7.2 & 15.6 & 1.5 \\
\hline $\begin{array}{l}\text { NEO-PI-R } \\
\text { MILLON }\end{array}$ & 3.6 & 8.9 & \\
\hline MILLON & 6.3 & 13.3 & 1.5 \\
\hline CUIDA & 18.0 & 42.2 & - \\
\hline PAI & 1.8 & 4.4 & - \\
\hline SCL.R-90 & 2.7 & 6.7 & - \\
\hline PROYECTIVOS & 1.8 & 4.4 & - \\
\hline TONI-2 & .9 & 2.2 & _ \\
\hline IA-TP & 1.8 & 4.4 & - \\
\hline IPDE & .9 & 2.2 & - \\
\hline BARRAT-BIS & 1.8 & 4.4 & - \\
\hline ISRA & 1.8 & 4.4 & - \\
\hline STAXI-II & 3.6 & 8.9 & - \\
\hline OAS & .9 & 2.2 & - \\
\hline FES WES & .9 & 2.2 & - \\
\hline RORSCHARCH & 1.8 & 4.4 & - \\
\hline \multicolumn{4}{|l|}{ GRAFICO-MOTOR } \\
\hline \multirow{2}{*}{\multicolumn{4}{|c|}{ Pruebas utilizadas con menores }} \\
\hline & & & \\
\hline PATA NEGRA & 1.8 & 4.4 & - \\
\hline HTP & 15.3 & 33.3 & 3.0 \\
\hline CBC INFANTIL & 1.8 & 4.4 & \\
\hline TAMAI & 9.9 & 17.8 & 4.5 \\
\hline ESFA & .9 & 2.2 & - \\
\hline HSPQ & 9 & 2.2 & - \\
\hline PROY̌ECTIVOS INFANTIL & 16.2 & 33.3 & 4.5 \\
\hline BASC & 2.7 & 6.7 & - \\
\hline ESPA 29 & .9 & 2.2 & - \\
\hline WISC-R & 1.8 & 4.4 & - \\
\hline CAT-H & 3.6 & 8.9 & - \\
\hline FIGURA COMPLEJA DE REY & 1.8 & 4.4 & - \\
\hline CDS DEPRESIÓN INFANTIL & 2.7 & 6.7 & 1.5 \\
\hline ESCALA BAYLEY & 1.8 & 2.2 & 3.0 \\
\hline MACI & 1.8 & & - \\
\hline CORMAN & 1.8 & 4.4 & - \\
\hline
\end{tabular}

Nota: IP: Informe Psicólogo privado. IEPS: Informe equipo psicosocial.

$* * * p<.001$

\section{Discusión}

El presente estudio realizado en los juzgados de familia nos permite deducir las siguientes cuestiones:

$1^{\text {a }}$ Hipótesis: Una primera constatación hallada, es un importante sesgo de los autores de los informes del estudio, hacia el género femenino analógicamente con la estructura demográfica de la profesión, en la cual, dos tercios de los profesionales privados indicaron tener formación específica forense, y de éstos una tercera parte con grado de doctor; no obstante, un tercio de los IP no indicaron poseer la formación específica forense.
Tabla 7

Procedimientos utilizados en las evaluaciones de la custodia de menores. Comparación entre el presente estudio y el de Bow y Quinnell (2002).

\begin{tabular}{|c|c|c|c|}
\hline \multirow[t]{2}{*}{ Procedimiento } & \multirow[t]{2}{*}{$\begin{array}{c}\text { Estudio de Bow y Quinnell } \\
\text { (2002) } \\
\% \text { mediante el procedimiento }\end{array}$} & \multicolumn{2}{|c|}{$\begin{array}{l}\text { Este estudio } \\
\% \text { mediante el } \\
\text { procedimiento }\end{array}$} \\
\hline & & IP & IEPS \\
\hline Entrevistó al padre & 100.0 & 75.0 & 89.4 \\
\hline Entrevistó a la madre & 100.0 & 55.6 & 93.9 \\
\hline Entrevistó a cada menor & 92.3 & 88.9 & 31.8 \\
\hline Pruebas a los progenitores & 90.4 & 57.8 & 4.5 \\
\hline $\begin{array}{l}\text { Observación del menor en } \\
\text { consulta }\end{array}$ & 82.7 & 40.0 & 3.0 \\
\hline Revisión del expediente & 78.8 & 73.3 & 100.0 \\
\hline Coordinación con terceros: & 75.0 & 17.8 & 71.2 \\
\hline Terapeutas (si procede) & 77.7 & (c) & (c) \\
\hline Otros (tutores...) & 73.1 & (c) & (c) \\
\hline Médicos (si procede) & 65.5 & 31.1 & 31.8 \\
\hline Profesores (si procede) & 62.0 & 28.9 & 48.5 \\
\hline Parientes & 51.9 & (c) & (c) \\
\hline Pruebas de los menores & 38.5 & 64.4 & 12.1 \\
\hline Visita domicilio & 34.6 & 2.2 & 0.0 \\
\hline Pruebas de otros & & & \\
\hline significantes (si procede) & 33.3 & 15.6 & 12.1 \\
\hline $\begin{array}{l}\text { Entrevista conjunta inicial } \\
\text { Adultos }\end{array}$ & 15.4 & $-{ }^{\mathrm{a}}$ & $-{ }^{\mathrm{a}}$ \\
\hline Test objetivos de & & & \\
\hline personalidad & 87.8 & 48.9 & 3.0 \\
\hline Inventarios de parentalidad & 44.9 & $42.2^{\mathrm{b}}$ & 0.0 \\
\hline $\begin{array}{l}\text { Test proyectivos de } \\
\text { personalidad }\end{array}$ & 40.8 & 8.8 & 0.0 \\
\hline $\begin{array}{l}\text { Escalas de evaluación } \\
\text { infantil }\end{array}$ & 30.6 & 0.0 & 0.0 \\
\hline Test de Inteligencia & 22.4 & 0.0 & 0.0 \\
\hline Pruebas de habilidades & 0.0 & 2.2 & 0.0 \\
\hline Menores & & & \\
\hline $\begin{array}{l}\text { Test proyectivos de } \\
\text { personalidad }\end{array}$ & 21.6 & 78.9 & 0.0 \\
\hline $\begin{array}{l}\text { Escalas de percepción del } \\
\text { menor de sus padres }\end{array}$ & 21.6 & 0.0 & 0.0 \\
\hline Test objetivos de & & & \\
\hline personalidad & 19.6 & 17.8 & 0.0 \\
\hline Test de Inteligencia & 11.8 & 4.4 & 0.0 \\
\hline Test de habilidades & 3.9 & 0.0 & 0.0 \\
\hline
\end{tabular}

Nota: IP: Informe Psicólogo privado. IEPS: Informe equipo psicosocial. Adaptación realizada por los autores, con autorización por cortesía de Bow y Quinnell. a Condición no estudiada en esta investigación. b En este estudio se trata del Test "Cuida".

$2^{\text {a }}$ Hipótesis: La mayoría de los IP y los IEPS mostraron una estructura completa de sus informes; incluyeron la competencia parental y el tipo de custodia recomendado; fueron escritos en un lenguaje claro y comprensible y estuvieron estructurados en apartados: se confirma así, la hipótesis, respecto a que los IEPS resultaron mejor estructurados en mayor medida que los IP, si bien podría explicarse al disponer los primeros de un protocolo interno y los segundos, probablemente, por adoptar criterios dispares respecto a las normativas tal como concluyó el estudio de Molina et al. (2012). Los IEPS y los IPCF realizaron en mayor medida más informes completos que los IPSF. Dos tercios de los profesionales firmantes contaban con formación específica forense.

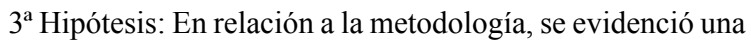
importante discordancia en relación a entrevistar a los menores: Los IP los entrevistaron de forma mayoritaria, mientras que los IEPS los entrevistaron de forma minoritaria, prescindieron de entrevistar a los menores bajo el argumento de evitar una posible victimización secundaria (Sataf, 2011). Basándose en esta suposición, los IEPS expusieron sus evaluaciones del núcleo familiar primario mediante los datos aportados por los progenitores y por coordinaciones con terceros. 
En relación al objeto de la pericia, se hallaron diferencias entre ambos grupos. Los IP evaluaron en mayor medida más traslados del lugar de residencia del menor, la presencia de un trastorno psicológico activo en un progenitor, o por la posibilidad de supuestos abusos sexuales, en tanto que los IEPS plasmaron mayor tasa de evaluación del régimen de visitas o de retirada de la potestad. En general, se incluyeron en promedio dos temas para evaluar. Si bien, los IEPS se ocuparon en mayor medida de la competencia parental, ergo la orden judicial, indicaba específicamente lo que el juez necesitaba saber. Por el contrario, los IP realizaron en mayor medida estudios sobre el tipo de custodia y la competencia parental, puesto que fue la demanda habitual proveniente de letrados y/o progenitores. Ambos progenitores acudieron a las entrevistas de los IEPS en más ocasiones que cuando fueron citados por los IP, porque procedían de requerimientos de los tribunales a los que no podían sustraerse; en cambio, los IP al ser contratados por una de las partes en litigio, la otra parte podía negarse a acudir para la evaluación pericial.

Los IPCF mostraron mayor tasa de estudio del proceso judicial; expusieron una mayor tasa los antecedentes de hecho; de la disolución de la pareja en mayor medida que los IPSF. Los IPCF mostraron mayor estructuración en el conjunto de las variables en mayor medida que los IPSF. Este resultado es sugerente de que la formación especializada mejora la calidad de los informes y respalda la necesidad de una exigencia de formación específica para intervenir como psicólogo forense (APA, 2013a; Molina et al., 2012; Jeffrey, Allen, Nancy, Andrew, Glenn, Jackie, y Nancy, 2009).

4 a Hipótesis: Contrariamente a lo esperado, sólo una cuarta parte de los informes reconocieron las limitaciones del mismo, pero los IEPS los hicieron en mayor medida que los IP. Aunque en líneas generales el análisis de los resultados concordó con los resultados tanto en los IP como en los IEPS, la comprobación de la veracidad del testimonio resultó muy escasa en su conjunto. Los IEPS contrastaron en mayor medida información aportada por terceros, y también refirieron más limitaciones del estudio. Más del 90\% de todos los informes respondieron a las cuestiones planteadas.

Los IEPS obtuvieron en mayor medida mejores puntuaciones que los IP en los contenidos de la evaluación. Dato que refuerza la importancia de seguir una metodología y estructuración tal como se recoge en las guías de buenas prácticas del COPM y COPC (Chacón et. al, 2009; COPC, 2014).

Alrededor de $2 / 3$ aportaron recomendaciones de guarda y de régimen de visitas: Para realizar recomendaciones de la guarda de menores y del régimen de visitas es indispensable entrevistar a todos los miembros de la unidad familiar, sin embargo, -en los tres grupos estudiados-, menos de un tercio, realizaron las entrevistas al conjunto familiar, aunque más de dos tercios optaron por recomendar la guarda y del régimen de visitas, aspecto divergente por cuanto que, si se han de realizar estas recomendaciones, es inexcusable examinar a todos los miembros de la unidad familiar, como se ha señalado reiteradamente en las guías orientadoras (APA, 2010a; APA, 2013a; APA, 2013b).

En el supuesto del informe parcial, en que sólo se evalúa la competencia parental, se debe limitar a recomendar si el progenitor evaluado dispone de aptitudes y/o actitudes adecuadas para la educación y cuidado del menor sin pronunciarse sobre la guarda, puesto que tales afirmaciones en los dictámenes sobre personas no evaluadas directamente conlleva el riesgo de presentar conclusiones erróneas, lo cual frecuentemente, ha sido objeto de debate en la literatura (Molina et al., 2012; EFPA, 2005).

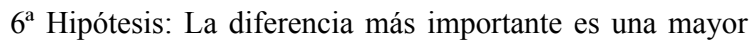
aplicación de pruebas psicométricas tanto en menores como en adultos, por parte de los IP en mayor medida respecto de los IEPS que apenas las utilizaron; el lenguaje utilizado fue comprensible sin utilizar jerga técnica; en todos los grupos no se expresó si detectaron la simulación en las evaluaciones. Hay una mayor diferencia en la aplicación de pruebas psicométricas tanto en menores como en adultos, por parte de los IP en mayor medida que los IEPS que apenas las utilizaron. Respecto a las pruebas psicométricas utilizadas encontramos una amplia gama, entre las que se destaca una preferencia mayoritaria por el test "CUIDA" y "MMPI" para adultos, y test proyectivos para menores y "TAMAI".

$7^{\text {a }}$ Hipótesis: Respecto a las diferencias en los procedimientos utilizados en las evaluaciones de la custodia de menores entre IP e IEPS y el estudio de Bow y Quinnell (2002), la primera evidencia que se observa del estudio de éstos últimos, es que entrevistan siempre ambos progenitores, y a los menores en una tasa superior al $90 \%$, aspecto que contrasta sustancialmente respeto a la polémica de evaluar o no, a los menores por parte del IEPS, y a la necesidad de evaluar al conjunto familiar cuando se trata de recomendar guarda y/o régimen de visitas del progenitor no custodio. Es destacable la gran diferencia que muestra el estudio de Bow y Quinnell (2002), al realizar el doble de observaciones en consulta que los IP y una diferencia mayor del $75 \%$ respecto del IEPS. Otro tanto, puede decirse de las visitas a domicilio, o de las pruebas psicométricas aplicadas.

Los datos extraídos del estudio avalan la necesidad de mayor formación y especialización en esta área relacionada con la protección de los menores. Son necesarias futuras investigaciones que permitan comparar los resultados obtenidos en este estudio, y que permitan profundizar las diferencias y similitudes entre los informes psicológicos privados e informes psicológicos de los equipos psicosociales, con datos reales y fiables sobre la estructura, metodología y contenidos de la ECM.

Se advierte una discusión, no resuelta por los peritos, que suscita importantes cuestiones: ¿cómo respetar el principio de intervención mínima en las evaluaciones de la custodia de menores, y a la vez prescindir de la fuente de información primaria que pueden ser los menores? Se puede tomar la referencia de la Sala de lo Civil del Tribunal Supremo (2013), que ordenó 
a la Audiencia Provincial de Orense a escuchar a los menores (TSJ, 2013). Otra cuestión que queda abierta es: ¿cuándo se consideran contraindicadas las exploraciones de menores, a fin de evitar, la victimización secundaria? ¿qué ponderación ganancia/pérdida se ha de considerar en este asunto, cuando la propia ley prevé la evaluación judicial del menor? ¿es mejor que la opinión de los menores se evalúe en la exploración judicial o por psicólogos expertos forenses? Por ello, se requiere nuevos estudios que valoren la existencia, y/o el impacto de una posible victimización secundaria que sea causada por la propia evaluación del menor. Aunque, de acuerdo con el Sataf (2011), se ha de respetar el principio de intervención mínima, el ISM, y valorar cuando la exploración del menor pueda estar contraindicada obtener dicha información mediante la coordinación en red con otros profesionales.

La importancia del presente estudio exploratorio radica en que es la primera vez que se ha realizado un estudio de esta naturaleza en nuestro país y se espera que promueva nuevos estudios que ayuden a mejorar la tarea de los informes periciales emitidos por psicólogos forenses, así como su aceptación por los Tribunales. También es importante subrayar que se elaboren más recomendaciones específicas como la mediación dado que se trata de familias en divorcios ad litem.

En relación a la formación, se observa, quizás reflejo de una progresiva concienciación, la necesidad de especialización para ejercer en esta área. Pero, resulta preocupante, que todavía un $35.6 \%$ de los psicólogos intervengan ante los tribunales de familia sin una adecuada formación específica forense.

Entre las limitaciones, se debe comentar que: a) se realizó en medio natural en el ámbito aplicado por cuanto presenta mayor validez externa y menor validez interna; b) no es posible asegurar la igualdad entre los grupos respecto a otras posibles variables extrañas; c) no es posible establecer relaciones causales; ni generalizar los resultados ya que es un estudio descriptivo; d) la muestra tiene una limitación territorial circunscrito a la ciudad de Barcelona; e) futuras investigaciones deberían ampliar el tamaño de la muestra.

\section{Referencias}

1. Aguilera, G. y Zaldívar, F. (2003). Opinión de los jueces (derecho penal y de familia) sobre el informe psicológico forense. Anuario de Psicología Forense 13, 95-122.

2. Alascio, L. y Marín, I. (2007). Juntos pero no revueltos: la custodia compartida en el nuevo art. 92 CC. In Dret, 3, 1-23.

3. Amato, P.R. (2000). The consequences of divorce for adults and children. Journal of Marriage and the Family, 62, 1269-1287. doi:10.1111/j.17413737.2000.01269.x

4. American Psychological Association (1994). Guidelines for child custody evaluations in divorce proceedings. American Psychologist, 49, 677-680. http://dx.doi.org/10.1037/0003066X.49.7.677

5. American Psychological Association (2010a). Ethical prin- ciples of psychologists and Code of Conduct. Washington: American Psychological Association.

6. American Psychological Association (2010b). Guidelines for child custody Evaluations in Family Law Proceedings. Washington: American Psychological Association, 65, 863867. http://dx.doi.org/10.1037/a0021250

7. American Psychological Association (2013a). Specialty guidelines for forensic psychology. American Psychologist, 68, 7-9. http://dx.doi.org/10.1037/a0029889

8. American Psychological Association (2013b). Guidelines for psychological evaluations in child protection matters. Washington: American Psychological Association,68, 20-31. http://dx.doi.org/10.1037/a0029891

9. Andersson, G. (2002). Children's experience of family disruption and family formation: Evidence from 16 FFS countries. Demographic Research, 7, 343-364. http://dx.doi. org/10.4054/DemRes.2002.7.7

10. Arce, R. y Fariña, F. (2005). Peritación psicológica de la credibilidad del testimonio, la huella psíquica y la simulación: el sistema de evaluación global (SEG). Papeles del Psicólogo, 26, 59-77.

11. Arce, R., Fariña, F. y Carballal, A. (2009). Creación y validación de un protocolo de evaluación forense de las secuelas psicológicas de la violencia de género. Psicothema, 21, 241-247.

12. Arce, R., Fariña, F. y Seijo, D. (2005). Razonamientos judiciales en procesos de separación. Psicothema, 17, 57-63.

13. Arch, M. (2010). Divorcio conflictivo y consecuencias en los hijos: implicaciones para las recomendaciones de guarda y custodia. Papeles del Psicólogo, 31, 183190.

14. Arch, M. y Jarne, A. (2010). Instrumentos de evaluación psicológica en las peritaciones de guarda y custodia de los niños: Uso y admisibilidad. Anuario de Psicología Jurídica, 20, 59-70. http://dx.doi.org/10.5093/jr2010v20a6

15. Austin, W. G. y Drozd, L. M. (2013). Judge's Bench Book for Application of the Integrated Framework for the Assessment of Intimate Partner Violence in Child Custody Disputes. Journal of Child Custody, 10, 99-119. http:// dx.doi.org/10.1080/15379418.2013.796850

16. Boletín Oficial del Estado. A-1990-31312. (1990). De 31 de Diciembre. Instrumento de Ratificación de la Convención sobre los Derechos del Niño, adoptada por la Asamblea General de las Naciones Unidas el 20 de noviembre de 1989.

17. Boletín Oficial del Estado A-2000-323. (2000). Ley 1/2000 de Enjuiciento Civil.

18. Boletín Oficial del Estado A-2010-13312. (2010). Ley 25/2010. Llibre $2^{\circ}$ Codi Civil de Catalunya.

19. Boletín Oficial del Estado A-1981-16216. (1981). Ley 30/1981, de 7 de Julio, por la que se modifica la regulación del matrimonio en el Código Civil y se determina el procedimiento a seguir en los casos de nulidad, separación 
y divorcio.

20. Bow, J. N. y Quinnell, F. A. (2001). Psychologist current practices and procedures in child custody evaluations: Five years after American psychological association guidelines. Professional Psychology: Research and Practice, 32, 261268. http://dx.doi.org/10.1037/0735-7028.32.3.261

21. Bow, J. y Quinnell, F.A. (2002). A critical review of child custody evaluations reports. Family Court Review, 40, 164176. http://dx.doi.org/10.1111/j.174-1617.2002.tb00827.x

22. Bow, J. y Quinnell, F.A. (2004). Critique of child custody evaluations by the legal profession. Family Court Review, 42, 115-127. http://dx.doi.org/10.1177/1531244504421009

23. Bow, J. N. (2006) Review of empirical research on child custody practice, Journal of Child Custody, 3, 23-50. http:// dx.doi.org/10.1300/J190v03n01 02

24. Chacón, F., García, J. F., Gómez, R. y Vázquez, B. (2009). Guía de buenas prácticas para la elaboración de informes psicológicos periciales sobre custodia y régimen de visita de menores. Madrid. Colegio Oficial de Psicólogos de Madrid. Recuperado de http:/www.infocop.es/view_article.asp? $\mathrm{id}=2462$

25. Colegio Oficial de Psicólogos de Cataluña. (2014). Guía de buenas prácticas para la evaluación psicológica forense y la práctica pericial. Barcelona. COPC. Disponible en http:// www.copc.org/Paginas/Ficha.aspx?IdMenu=E5406E2D6EDB-48D9-8026-30CCB62B7220.

26. De Torres, J. M. (2009). Interés del menor y Derecho de familia. Una perspectiva multidisciplinar. Madrid: Iustel.

27. De Torres, J. M. (2011). Custodia compartida: Una alternativa exigida por la nueva realidad social. InDret, 4, 1-61. Recuperado en 14-06-2013, de http://www.indret.com/ pdf $/ 853$ es.pdf.

28. Del Río, C. (2000). Informes de parte en conflictos matrimoniales: implicaciones deontológicas. Infocop, 10, 15-20.

29. Domínguez, R., Gamero, M., González, M. y Roca, A. (2009). Impacte dels informes del SATAF en les resolucions judicials. Efectes dels informes emesos pels equips psicosocials de Catalunya en les mesures judicials adoptades. Documents de Treball. Centre d'Estudis Jurídics $i$ Formació Especialitzada. Generalitat de Catalunya.

30. Echeburúa, E., Muñoz, J. M. y Loinaz, I. (2011). La evaluación psicológica forense frente a la evaluación clínica : propuestas y retos de futuro. International Journal of Clinical and Health Psychology, 11, 141-159.

31. European Federation of Psychologists Associations (2005). Metha code of ethics. Recuperado de http://ethics.efpa.eu/ meta-code/

32. Erard, R. E. (2007). Picking cherries with blinders on: a comment on Erickson Et Al. (2007) Regarding the use of tests in family court. Family Court Review, 45, 175-184. http://dx.doi.org/10.1111/j.1744-1617.2007.00137.x

33. Gardner, R.A. (1998). The parental alienation syndrome (Vol. $2^{\circ}$ Ed.). Cresskill, N.J., US: Creative Therapeutics Inc.
34. Gould, J. W. y Martindale, D. A. (2007). The art and science of child custody evaluations. New York: Guilford. Greenberg.

35. Horvath, L. S., Logan, T. K. y Walker, R. (2002). Child custody cases: A content analysis of evaluations in practice. Professional Psychology: Research and Practice, 33, 557565. http://dx.doi.org/10.1037/0735-7028.33.6.557

36. Instituto Nacional de Estadística. (2013). Estadística de nulidades, separaciones y divorcios. Madrid: INE.

37. Jeffrey, Z., Allen K. H., Nancy A. M., G. Andrew H. B., Glenn A. A., Jackie K. G. y Nancy, K.B. (2009). Ethical and professional considerations in divorce and child custody cases. Professional Psychology: Research and Practice, 40, 539-549. http://dx.doi.org/10.1037/a0017853

38. Kuehnle, K., Greenberg, L. R. y Gottlieb, M. C. (2004). Incorporating the principles of scientifically based child interviews into family law cases. Journal of Child Custody, 1, 97-114. http://dx.doi.org/10.1300/J190v01n01 07

39. Manzanero, A. L. y Muñoz, J. (2011). La prueba pericial psicológica sobre la credibilidad del testimonio: Reflexiones psico-legales. Madrid: SEPIN.

40. Martindale, D. A., Martín, L., Austin, W. G., Drotd, L., Gould-Saltman, G., Kirpatrck, H. D. y Stahl, P. M. (2007). Model standars of practice for child custody evaluation. Family Court Review, 45, 70-91. http://dx.doi.org/10.1111/ j.1744-1617.2007.129 3.x

41. Molina, A., Arch, M. y Jarne, A. (2012). Conocimiento y aplicación de los Principios Éticos y Deontológicos por Parte de los Psicólogos Forenses Expertos en el Ámbito de Familia. Anuario de Psicología Jurídica, 22, 77-93. http:// dx.doi.org/10.5093/aj2012a8

42. Muñoz, J.M. (2010). El constructo Síndrome de Alienación Parental (SAP) en Psicología Forense: Una propuesta de abordaje desde la evaluación pericial psicológica. Anuario de Psicología Jurídica, 20, 5-14. http://dx.doi.org/10.5093/ jr2010v20a2

43. O.N.U. (20 de Noviembre de 1989). Convención de las Naciones Unidas sobre los Derechos del Niño. Ginebra: ONU.

44. Ramírez, M. (2003). Cuando los padres se separan. Alternativas de custodia. Guía Práctica. Madrid: Pirámide.

45. Servei d'Assessorament Tècnic en l'Àmbit de Família (2010). Programa d'Assessorament en l'Àmbit de Família. Departament de Justícia. Generalitat de Catalunya.

46. Servei d'Assessorament Tècnic en l'Àmbit de Família (2011). Guia d'intervenció tècnica en menors. Barcelona: Departament de Justícia. Generalitat de Catalunya.

47. Tippins, T. y Wittmann, J. P. (2005). Empirical and ethical problems with custody recommendations: a call for clinical humility and judicial vigilance. Family Court Review 43, 193-222. http://dx.doi.org/10.1111/j.17441617.2005.00019.x

48. Tribunal Supremo de Justicia (2013). Resolución de la Sala 
de lo Civil, por la que ordena a la Audiencia Provincial de Orense a escuchar a los dos hijos de una pareja antes de decidir si concede el régimen de custodia compartida $o$ se la otorga a uno de los progenitores. Recuperado el 20-12-2014, de http:/www.aranzadi.es/actualidad/noticias/ el-tribunal-supremo-ordena-un-juez-escuchar-dos-menores-para-resolver-sobre-el

Fecha de recepción: 2 de septiembre, 2014

Fecha de recepción de la primera versión modificada: 6 de noviembre

de 2014

Fecha de recepción de la segunda versión modificada: 25 de diciembre

de 2014

Fecha de aceptación: 12 de marzo de 2015 